\title{
How repulsive can a pairing interaction be?
}

\author{
Quantum phase transition in a clean superconductor with repulsive \\ dynamical interaction
}

Authors: Dimitri Pimenov and Andrey V. Chubukov

arXiv:2112.06273

\section{Recommended with a Commentary by Jörg Schmalian, Karlsruhe Institute of Technology}

The attractive interaction between electrons that is mediated by phonons is at the heart of the Bardeen-Fröhlich model and the effective Hamiltonian of the BCS theory of superconductivity. An immediate concern is that this interaction should we outweighed by the repulsive Coulomb interaction. Two important insights clarified this issue to a great extent. On the one hand repulsive interactions renormalize in the pairing channel to smaller values until one reaches the frequency regime of attractive phonons, at least for phonon frequencies smaller than the Fermi energy[1]. In addition, Bardeen and Pines[2] showed that the repulsive interaction is significantly reduced by screening effects of the combined electron-phonon problem

$$
V_{0}(\boldsymbol{q})=\frac{4 \pi e^{2}}{q^{2}} \rightarrow V(\boldsymbol{q}, \omega)=\frac{4 \pi e^{2}}{q^{2} \varepsilon(\boldsymbol{q}, \omega)},
$$

with dielectric constant $\varepsilon(\boldsymbol{q}, \omega)$. In its simplest form of the jellium model holds[3]

$$
\frac{1}{\varepsilon(\boldsymbol{q}, i \omega)}=\frac{q^{2}}{q^{2}+q_{\mathrm{TF}}^{2}}\left(1-\frac{\Omega_{q}^{2}}{\Omega_{q}^{2}+\omega^{2}}\right),
$$

with Thomas-Fermi momentum $q_{\mathrm{TF}}^{2}$ and acoustic phonon frequency $\Omega_{q} \sim q$. Within the Eliashberg formalism, the sign of $V(\boldsymbol{q}, i \omega)$ at imaginary frequencies is the most direct criterion for a dynamic interaction to be attractive or repulsive. The importance of such retarded interactions was discussed early on in the theory of superconductivity[4] with recent applications and generalizations to describe pairing in e.g. $\mathrm{SrTiO}_{3}$ and $\mathrm{Bi}[5]$. It turns out that $\varepsilon(\boldsymbol{q}, i \omega)>0$ still allows for $s$-wave superconductivity, albeit with a pairing potential $\Delta(i \omega)$ that changes sign as function of $\omega[6,7]$.

Pimenov and Chubukov address two natural question that could have been discussed long ago: i) How repulsive can such an interaction be to still yield a superconducting ground state? ii) How does superconductivity disappear for sufficiently strong repulsive dynamic interaction? To proceed they average the Eliashberg equations over momenta which yields the usual result

$$
\Delta(i \omega)=-\rho_{F} \int_{-E_{F}}^{E_{F}} d \omega^{\prime} \frac{V\left(i \omega-i \omega^{\prime}\right) \Delta\left(i \omega^{\prime}\right)}{\sqrt{\omega^{\prime 2}+\Delta\left(i \omega^{\prime}\right)^{2}}}
$$


and consider the retarded interaction

$$
V(i \omega)=V_{0}\left(f-\frac{\Omega_{0}^{2}}{\Omega_{0}^{2}+\omega^{2}}\right)
$$

at weak coupling $\left(\rho_{F} V_{0} \ll 1\right)$ and for variable $f$. $E_{F}$ is of the order of the Fermi energy and $\rho_{F}$ the density of states at the Fermi level.

For $f<1$ it holds $V(i \omega)<0$ and superconducting solutions exist by virtue of the usual Cooper instability. However, a fully repulsive interaction still allows for solutions if $f<f_{c} \approx$

$1+\rho_{F} V_{0}\left(2 \log \frac{E_{F}}{\Omega_{0}}-1\right)$. This is realized through the sign changing gap function $\Delta(i \omega)$. The position $\omega_{0}$ of the node $\Delta\left(i \omega_{0}\right)=0$ vanishes like $\omega_{0} \sim\left(f_{c}-f\right)^{1 / 2}$, while $\Delta$ is exponentially small $\sim \exp \left(-A /\left(f_{c}-f\right)\right)$. For $f>f_{c}$ the ground state is non-superconducting. Hence, one arrives at a new superconducting quantum critical point (QCP).

The most peculiar behavior is associated with phase fluctuations near this superconducting critical point. If compared to the much-studied Abrikosov-Gor'kov QCP that occurs due to pair-breaking impurity scattering, the superfluid stiffness $n_{s}$ does not vanish as $f \rightarrow f_{c}$. Instead, it jumps discontinuously at the transition. For $f>f_{c}$ superconductivity disappears and $n_{s}$ must, of course, vanish. On the other hand, for $f \rightarrow f_{c}-0^{+}$the longest wavelength electrodynamics is similar to the one in the usual BCS limit with $n_{s}$ given by the particle number. Pimenov and Chubukov however show that the behavior is much richer. If one considers the stiffness at finite momenta $n_{s}(\boldsymbol{q})$ it becomes a scaling function of $\xi q$ with coherence length $\xi=v_{F} / \Delta(0)$ that diverges exponentially at the transition. Even though $n_{s}$ jumps in the $q \rightarrow 0$ limit, it is suppressed for $q>\xi^{-1}$ and therefore vanishes continuously at any finite $q$. Hence regions inside the coherence volume have strong phase fluctuations and become fragile, while the system acquires a global stiffness as robust as any BCS superconductor. The rigidity of the pairing state is undermined from within. For a two-dimensional system, this transition should be reachable by changing the dielectric constant of a substrate or by varying a gate voltage, making these results rather relevant. Thus far we have an understanding of the mean-field behavior of this transition. It is a very interesting problem to develop the theory of critical fluctuations and the universality class for this new superconducting QCP that may affect the normal state nearby.

\section{References}

[1] P. Morel and P. W. Anderson, Phys. Rev. 125, 1263 (1962).

[2] J. Bardeen and D. Pines, Phys. Rev. 99, 1140 (1955).

[3] P. G. de Gennes, Superconductivity of metals and alloys, Addison-Wesley, NY, (1989).

[4] V. Gurevich, A. Larkin, Y. A. Firsov, Sov. Phys.-Solid State 4, 131 (1962).

[5] J. Ruhman and P. A. Lee, Phys. Rev. B 94, 224515 (2016); ibid. 96, 235107 (2017).

[6] W. L. McMillan, Phys. Rev. 167, 331 (1968).

[7] H. Rietschel and L. J. Sham, Phys. Rev. B 28, 5100 (1983). 